\title{
Role of Increased Lipoprotein (a) in Retinal Vein Occlusion: A Systematic Review and Meta- analysis
}

\author{
Francesco Paciullo ${ }^{1}$ David Giannandrea ${ }^{2 \odot}$ Gianni Virgili $^{3}$ Carlo Cagini ${ }^{4}$ Paolo Gresele ${ }^{1}$
}

${ }^{1}$ Division of Internal and Cardiovascular Medicine, Department of Medicine and Surgery, University of Perugia, Perugia, Italy

2 Division of Neurology and Stroke Unit, Department of Neurology, Gubbio and Città di Castello Hospital, Perugia, Italy

3 Department of Neurosciences, Psychology, Drug Research and Child Health (NEUROFARBA), Ophthalmology Clinic, University of Firenze and AOU Careggi, Florence, Italy

${ }^{4}$ Section of Ophthalmology, Department of Medicine and Surgery, University of Perugia, Perugia, Italy

TH Open 2021;5:e295-e302.

\author{
Address for correspondence Paolo Gresele, MD, PhD, Division of \\ Internal and Cardiovascular Medicine, Department of Medicine, \\ University of Perugia, Strada Vicinale Via delle Corse, 06126 Perugia, \\ Italy (e-mail: paolo.gresele@unipg.it).
}

\begin{abstract}
Keywords

- cardiovascular risk

- retinal vein occlusion

- unusual site vein thrombosis

Background Increased lipoprotein (a) [LP(a)] has been associated with enhanced risk of cardiovascular events and more recently with venous thromboembolism. However, there is inconclusive data on the association between enhanced $L p(a)$ and retinal vein occlusion (RVO). We aimed to assess the role of $L p(a)$ in RVO.

Methods We performed a systematic review and meta-analysis of the studies addressing the role of $L p(a)$ in RVO. A systematic literature search was performed to identify all published papers reporting $L p(a)$ levels. Main outcome measures consisted of $L p(a)$ levels in patients with (cases) or without (controls) RVO.

Results We included 13 studies for a total of 1,040 cases and 16,648 controls. Lp(a) levels above normal limits were associated with RVO (OR 2.38, 95\% Cl 1.7-3.34) and patients with RVO had higher $\mathrm{LP}(\mathrm{a})$ levels than controls (weighted mean difference: $13.4 \mathrm{mg} / \mathrm{dL}, 95 \% \mathrm{Cl} 8.2-18.6)$.

Conclusion Increased LP(a) levels associate with RVO and should be included among diagnostic and prognostic indexes for this unusual-site vein thrombosis. Therapeutic interventions aimed to lower $L p(a)$ should be tested in RVO patients.
\end{abstract}

\section{Introduction}

Retinal vein occlusion (RVO) is due to the thrombotic obstruction of retinal veins. Affecting 16 million people worldwide, RVO is the second most common retinal disease after diabetic retinopathy and it may be associated with serious consequences such as neurovascular glaucoma, retinal detachment, and ultimately blindness. ${ }^{1}$ Based on the site of vascular occlusion RVO is distinguished in central retinal

received

November 17, 2020

accepted after revision

February 26, 2021
DOI https://doi.org/

$10.1055 / \mathrm{s}-0041-1732803$.

ISSN 2512-9465. vein occlusion (CRVO), located in the central retinal vein at the passage through the lamina cribrosa, branch retinal vein occlusion (BRVO), involving one of the branches of the central retinal vein at an arteriovenous crossing, and hemispheric retinal vein occlusion (HRVO), involving the venous return from approximatively one half of the retina. BRVO is four times more common than CRVO, while bilateral vein thrombosis is very rare. ${ }^{1}$ BRVO usually manifests as a sudden painless decrease in vision or a visual field defect, while 
CRVO usually presents with sudden, unilateral, painless loss of vision. ${ }^{2}$ Unlike other vein thromboses, thrombophilia does not seem to play a major role in RVO, a conclusion supported by a recent meta-analysis which questioned the role of thrombophilia in retinal arterial and venous occlusive disease. ${ }^{3}$ On the other hand, several common cardiovascular risk factors, such as hypertension, diabetes, and hyperlipemia, were reported to be predisposing factors for $\mathrm{RVO}^{4}$ and to enhance the risk of RVO recurrence. ${ }^{2,4}$ These findings suggest that although RVO is a venous thrombosis it has more characteristics in common with atherosclerosis than with venous thromboembolism (VTE). ${ }^{3-5}$ Accordingly, despite some evidence of efficacy, ${ }^{6}$ anticoagulation is seldom administered to these patients and it is mostly reserved to younger subjects with acute occlusion, while cardiovascular risk factors control is the most widely used and strongly recommended measure to prevent RVO recurrence. ${ }^{2}$ Because of these uncertainties, medical management of this frequent and disabling condition is still far from optimal and a step forward in the knowledge of RVO pathogenesis is strongly required to identify appropriate therapeutic targets.

Lipoprotein (a) [Lp (a)] is a complex lipoprotein involved in tissue repair and wound healing. ${ }^{7} \mathrm{Lp}(\mathrm{a})$ resembles structurally low density lipoproteins (LDLs) from which it differentiates for the presence of apolipoprotein (a) [(apo(a)], a complex glycoprotein covalently bound to apo(b) by a disulfide bond. ${ }^{8}$ Due to its high affinity for glycosaminoglycans of the human arterial wall, even higher than that of LDL, Lp(a) easily accumulates in the intima of large and medium size arteries where it promotes monocyte and macrophage recruitment and activates a local inflammatory response favoring atheroma development and finally arterial thrombosis. ${ }^{9}$ Moreover, its apo(a) moiety competes with plasminogen, with which it shares more than $80 \%$ structural homology, thus exerting an antifibrinolytic effect. ${ }^{7,8}$ Finally, Lp(a) promotes platelet aggregation through mechanisms incompletely understood, induces the synthesis of plasminogen activator inhibitor, and depresses the synthesis of tissue factor pathway inhibitor in all enhancing blood coagulation. ${ }^{7,8,10}$ Unfortunately, even if some therapies have been described to lower $\mathrm{L}(\mathrm{a})$ levels, no specific treatment is still available to manage hyper-lp(a)-lipoproteinemia. Mendelian randomization studies confirmed a wide, genetically determined interindividual variation of $\operatorname{Lp}(a)$ circulating levels and molecular dimensions. ${ }^{9}$ Interestingly, the small dimension $\mathrm{Lp}(\mathrm{a})$ phenotype, the most proatherogenic, is usually associated with high plasma levels configuring a high CV risk profile. $^{11,12}$ Indeed, large population studies and metaanalyses indisputably showed that increased $L p(a)$ levels are associated with cardiovascular events. ${ }^{12}$ In particular, a strong correlation between $\mathrm{Lp}(\mathrm{a})$ levels and myocardial infarction or stroke risk has been shown in several prospective and retrospective population studies. ${ }^{13-19}$ On the contrary, the role of Lp(a) in VTE is less clear. ${ }^{20,21}$ In fact, while a recent systematic review and meta-analysis including 14 studies for a total of 14,000 patients concluded that Lp (a) levels associate with increased risk of VTE, ${ }^{20}$ a subsequent prospective study from the Kuopio Ischemic Heart Disease cohort in 2,180 men followed for a median period of 24.9 years resolved the opposite. ${ }^{21}$ Therefore, the association between $\mathrm{Lp}(\mathrm{a})$ and VTE remains controversial.

Concerning RVO, several prospective and retrospective cohort studies have reported an association between elevated $L p(a)$ levels and retinal vessel occlusive disease. ${ }^{22-34}$ However, due to the small number of patients enrolled in the individual studies, the exact role of $\operatorname{Lp}(\mathrm{a})$ in RVO risk remains uncertain. Aim of the present study was to carry out a systematic review and meta-analysis of the studies evaluating the association between $\mathrm{Lp}(\mathrm{a})$ levels and RVO incidence.

\section{Methods}

This systematic review and meta-analysis was performed following the PRISMA guidelines (www.prisma-statement. org) and it has been submitted to the International Prospective Register of Systematic Reviews (PROSPERO) (ID: 196552). Search strategies, methods for study quality assessment, and statistical plan were established a priori as well as the inclusion criteria and outcomes.

\section{Search Strategy}

We performed an electronic search through the Scopus, PubMed, and Google Scholar databases using the keywords "retinal vein occlusion" OR "RVO" OR "retinal vein thrombosis" and "lipoprotein (a)" OR "Lp(a)," without data or language restrictions, up to September 21, 2020. The titles, abstracts, and full text of all retrieved documents were carefully evaluated, and the reference list of all papers was examined to extract articles of potential interest and those reporting data on $\mathrm{Lp}(\mathrm{a})$ levels in RVO were included in the analyzed literature.

\section{Study Selection and Data Extraction}

Study selection was independently made by two reviewers (F.P. and D.G.) and disagreements were solved through discussion and when required with the opinion of a third investigator (P.G.). All case-control studies on patients with CRVO or BRVO reporting Lp(a) plasma levels were considered eligible for analysis with no restrictions about gender or age. Gray literature, or evidence not published in commercial publications was included in the systematic review. Data on arterial retinal occlusion, when available were not included in the analysis. Case reports were not included.

\section{Statistical Analysis and Risk of Bias Assessment}

A meta-analysis was carried to calculate the individual and pooled odds ratios (ORs) and their relative 95\% confidence intervals $(95 \% \mathrm{CI})$. The analysis was performed using Review Manager (Version 5.4). A random effect model was applied to evaluate the ORs of the association between high $\mathrm{Lp}(\mathrm{a})$ levels and RVO. Z-scores were used to test the overall effect with $p<$ 0.05 for significance. Results were presented with $95 \%$ CI. $I^{2}$ statistic and the Chi-square Cochrane $Q$ test were performed to evaluate statistical heterogeneity, which was considered significant when $p<0.1$. Attributable risk fraction was 


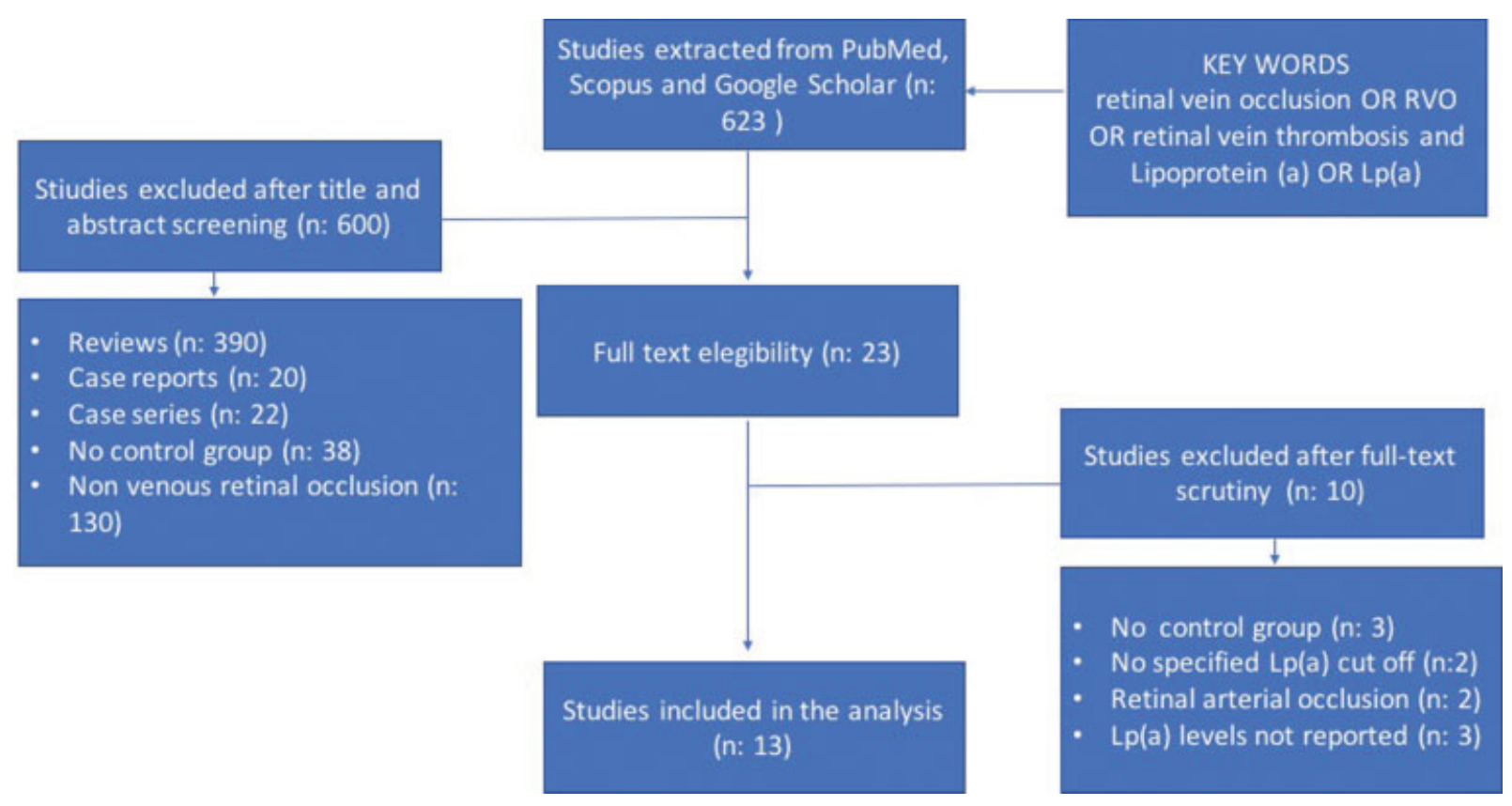

Fig. 1 Search strategy and study selection.

calculated as $[\mathrm{P}(\mathrm{RR}-1) / \mathrm{P}(\mathrm{RR}-1)+1]$, where $P=$ prevalence of risk factor in the population and RR is the relative risk. ${ }^{35} \mathrm{Lp}$ (a) levels were expressed as $\mathrm{mg} / \mathrm{dL}$. Publication bias was graphically analyzed by funnel plot. When $\operatorname{Lp}(a)$ data were reported as medians or means, a weighted mean difference (WMD) was calculated, and sample means and standard deviations were estimated and data meta-analyzed. When $\mathrm{Lp}(\mathrm{a})$ levels were expressed as medians and IQR, means and SD were estimated as previously described. ${ }^{36,37}$ In the study of Gumus et al, RVO Lp(a) mean levels and SD were obtained from the mean levels and SD of two groups (BRVO and CRVO). To avoid possible bias related to the variability of measures among studies, a random effect model was applied. Similarly, a random effect model was used to evaluate the association between $\mathrm{Lp}(\mathrm{a})$ plasma levels and RVO.

\section{Results}

Out of 623 articles initially retrieved by our search strategy (35 Scopus, 556 Google Scholar, 32 PubMed), 610 were excluded because of reviews or case reports, studies not reporting $\operatorname{Lp}(\mathrm{a})$ plasma levels or studies in patients affected by retinal arterial thrombosis ( - Fig. 1 ). At the end 13 studies, for a total number of 1,040 cases and 16,648 controls, were included in the analysis. ${ }^{22-34}$ The characteristics of the included studies are summarized in - Table 1. Quality assessment of the studies was performed according to the criteria suggested by the Newcastle-Ottawa scale $^{38}$ (-Table 2, - Supplementary Table S1). Despite the nonprospective nature of the included studies, with only observational and in most cases cross-sectional studies, the overall quality was considered satisfactory being high or intermediate for all but one paper. Association between RVO risk and Lp (a) values was assessed in 10 studies $^{22-25,27-32}$ for a total of 837 cases and 16,129 controls. For the study by KuhliHattenbach et al published in $2017^{22}$ only patients aged $>60$ years were considered to avoid possible duplicates with results of a second study from the same authors published later. ${ }^{28}$ Two studies by Glueck ${ }^{31,32}$ were included because the prospective nature of the second one $e^{32}$ contrarily to the observational nature of the first one excluded the possibility of duplicate cases. The $\mathrm{Lp}(\mathrm{a})$ plasma level cut-off values used for our analysis were chosen according to the normality ranges reported in 10 of the included studies (upper limit $35 \mathrm{mg} / \mathrm{dL}$ in two, $30 \mathrm{mg} / \mathrm{dL}$ in $6,20 \mathrm{mg} / \mathrm{dL}$ in one, and 10 $\mathrm{mg} / \mathrm{dL}$ in one). Prevalence of RVO was significantly higher in subjects with $\mathrm{Lp}(\mathrm{a})$ above upper limits compared with subjects within normal range (OR 2.38, 95\% Cl 1.7-3.34) (-Fig. 2). Heterogeneity among studies was not significant $\left(I^{2}=35 \%\right.$; Chi-square $\left.=13.93, p=0.12\right)(-$ Fig. 3$)$. The presence of publication bias suggested by the asymmetry of funnel plot was confirmed by the Peters test ${ }^{39}$ (-Supplementary Fig. S1). Lp(a)-attributable OVR risk-fraction was estimated to be $44 \%$. Mean or median values of $\mathrm{Lp}(\mathrm{a})$ in subjects with RVO versus controls were reported in four studies $^{26,33,34}$ for a total of 223 cases and 539 controls. Lp(a) levels were significantly higher in patients with RVO than in controls (WMD $13.4 \mathrm{mg} / \mathrm{dL}, 95 \%$ CI 8.2-18.6). Heterogeneity among studies was significant $\left(I^{2}=57 \%\right.$, Chi-square $=7$, $p=0.07$ ) (-Fig. 4).

\section{Discussion}

RVO is an unusual site vein thrombosis associated with potentially serious adverse outcomes, including blindness. In terms of predisposing factors RVO is closer to arterial 


\begin{tabular}{|c|c|c|c|c|c|c|c|c|c|c|c|c|c|}
\hline & 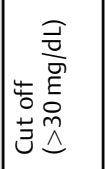 & 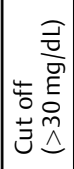 & 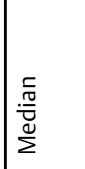 & 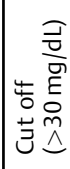 & 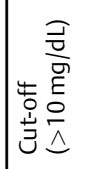 & 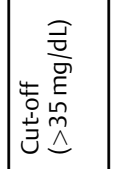 & 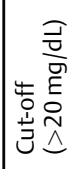 & 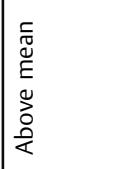 & $\mid \begin{array}{l}\frac{\bar{\sigma}}{\bar{\sigma}} \\
\frac{\tilde{\sigma}}{\tilde{\nu}}\end{array}$ & 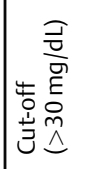 & 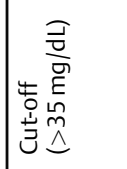 & 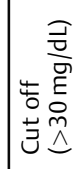 & 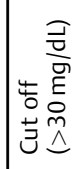 \\
\hline 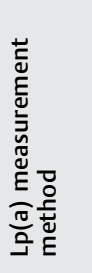 & 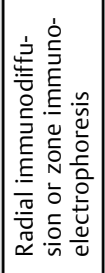 & 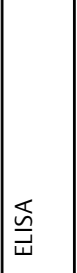 & 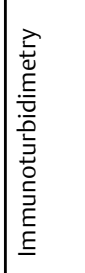 & 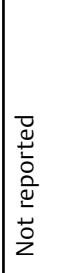 & 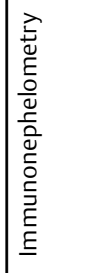 & 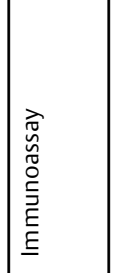 & $\mid \begin{array}{l}\widetilde{x} \\
\bar{\Psi}\end{array}$ & 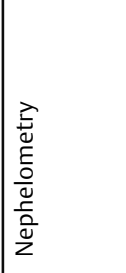 & 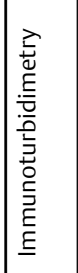 & 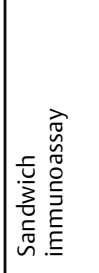 & 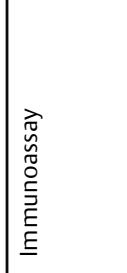 & 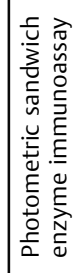 & 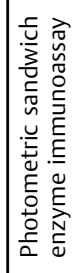 \\
\hline $\begin{array}{l}\frac{̃}{N} \\
\frac{N}{0} \\
\frac{0}{\tilde{E}} \\
\text { ñ }\end{array}$ & 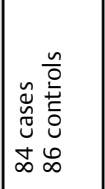 & 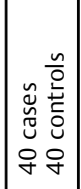 & 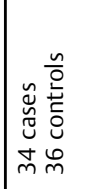 & 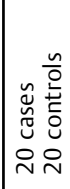 & 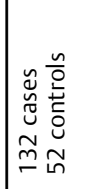 & 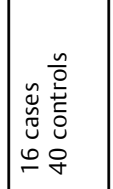 & 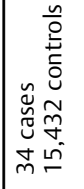 & 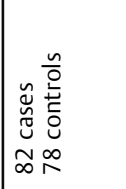 & 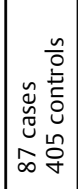 & 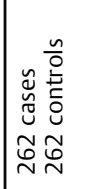 & 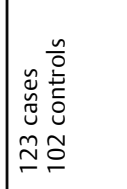 & 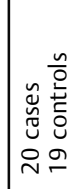 & 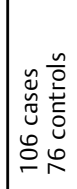 \\
\hline 点 & 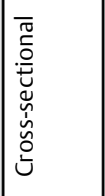 & 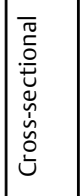 & 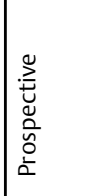 & 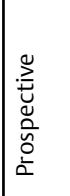 & 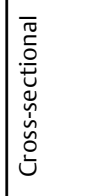 & 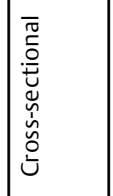 & 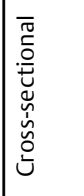 & 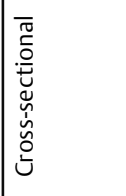 & 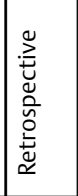 & 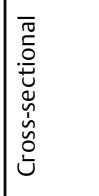 & 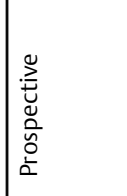 & 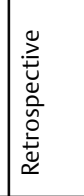 & 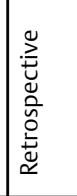 \\
\hline 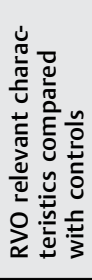 & 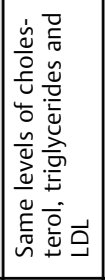 & 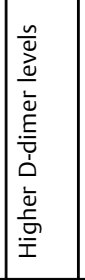 & 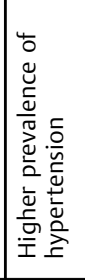 & 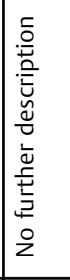 & 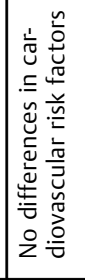 & 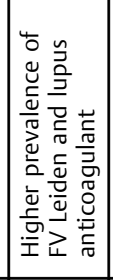 & 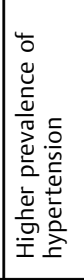 & 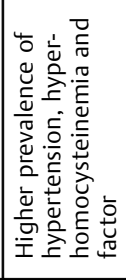 & 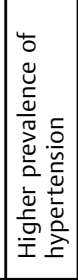 & 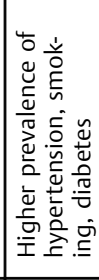 & 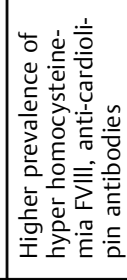 & 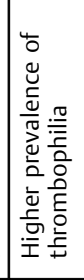 & 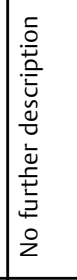 \\
\hline
\end{tabular}

\begin{tabular}{|c|c|c|c|c|c|c|c|c|c|c|c|c|c|}
\hline 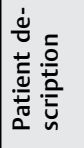 & 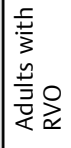 & 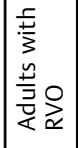 & 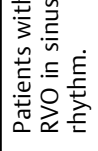 & 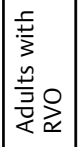 & 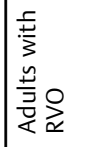 & 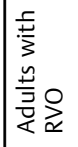 & 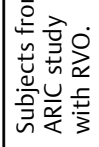 & 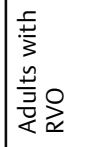 & 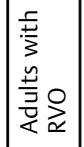 & 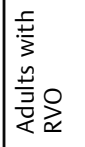 & 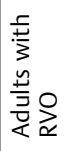 & 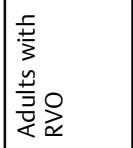 & \begin{tabular}{|l|}
$\frac{5}{3}$ \\
$\frac{5}{3}$ \\
$\frac{n}{3}$ \\
$\frac{3}{4}$
\end{tabular} \\
\hline 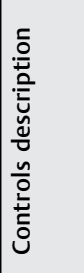 & 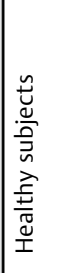 & 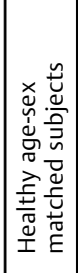 & 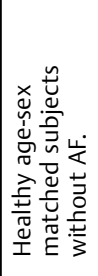 & 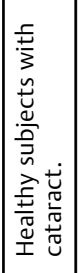 & 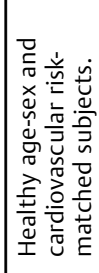 & 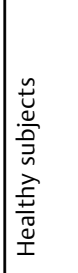 & 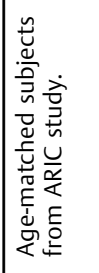 & 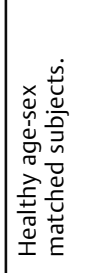 & 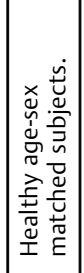 & 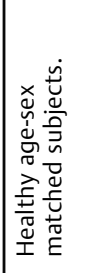 & 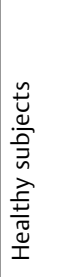 & 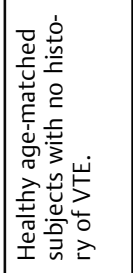 & 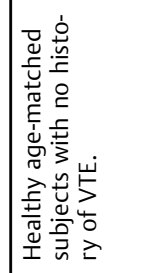 \\
\hline 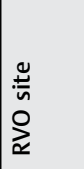 & 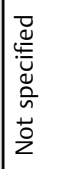 & 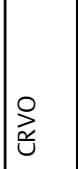 & 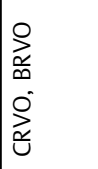 & 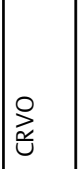 & 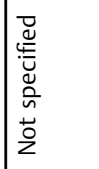 & 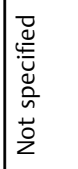 & 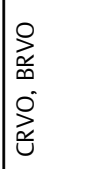 & 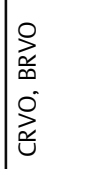 & 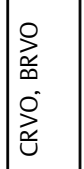 & 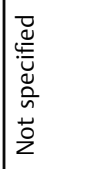 & 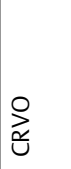 & 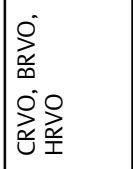 & 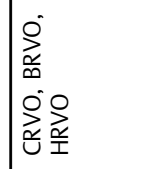 \\
\hline 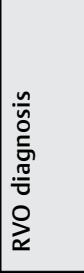 & 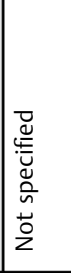 & 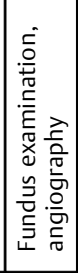 & 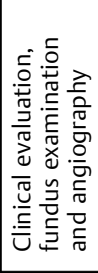 & 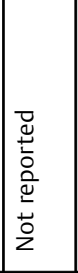 & 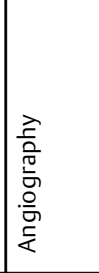 & 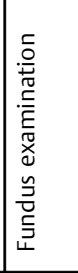 & 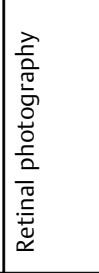 & 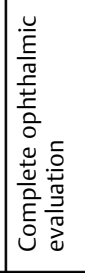 & 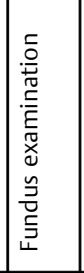 & 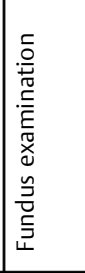 & 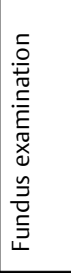 & 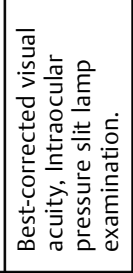 & 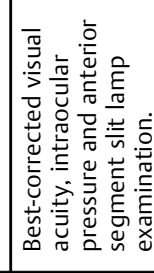 \\
\hline 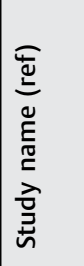 & 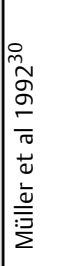 & 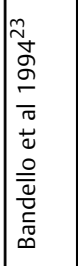 & 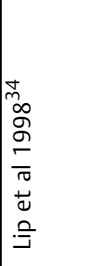 & 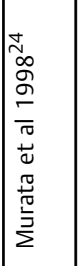 & 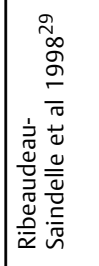 & 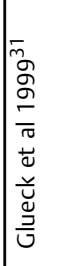 & 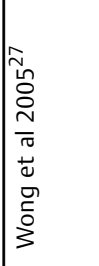 & 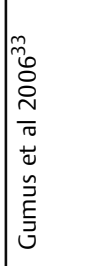 & 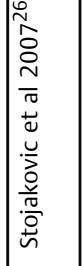 & $\mid$ & 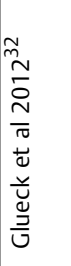 & 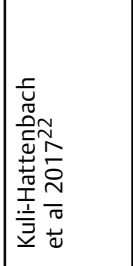 & 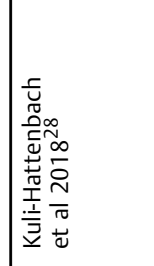 \\
\hline
\end{tabular}

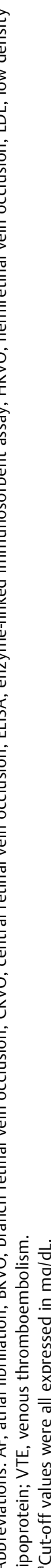


Table 2 Quality assessment of the studies included in the analysis

\begin{tabular}{|c|c|c|c|}
\hline Study name & Language & Year & New Ottawa Scale \\
\hline Müller et a ${ }^{30}$ & English & 1992 & Intermediate \\
\hline Bandello et $\mathrm{al}^{23}$ & English & 1994 & High \\
\hline Lip et $a^{34}$ & English & 1998 & Low \\
\hline Murata et $\mathrm{al}^{24}$ & English & 1998 & Intermediate \\
\hline Ribeaudeau-Saindelle et a ${ }^{29}$ & French & 1998 & Intermediate \\
\hline Glueck et al ${ }^{31}$ & English & 1999 & High \\
\hline Wong et $\mathrm{al}^{27}$ & English & 2005 & High \\
\hline Gumus et $\mathrm{al}^{33}$ & English & 2006 & High \\
\hline Stojakovic et al ${ }^{26}$ & English & 2007 & High \\
\hline Sofi et $\mathrm{al}^{25}$ & English & 2010 & High \\
\hline Glueck et al ${ }^{32}$ & English & 2012 & Low \\
\hline Kuli-Hattenbach et a ${ }^{22}$ & English & 2017 & High \\
\hline Kuli-Hattenbach et a ${ }^{28}$ & German & 2018 & Low \\
\hline
\end{tabular}

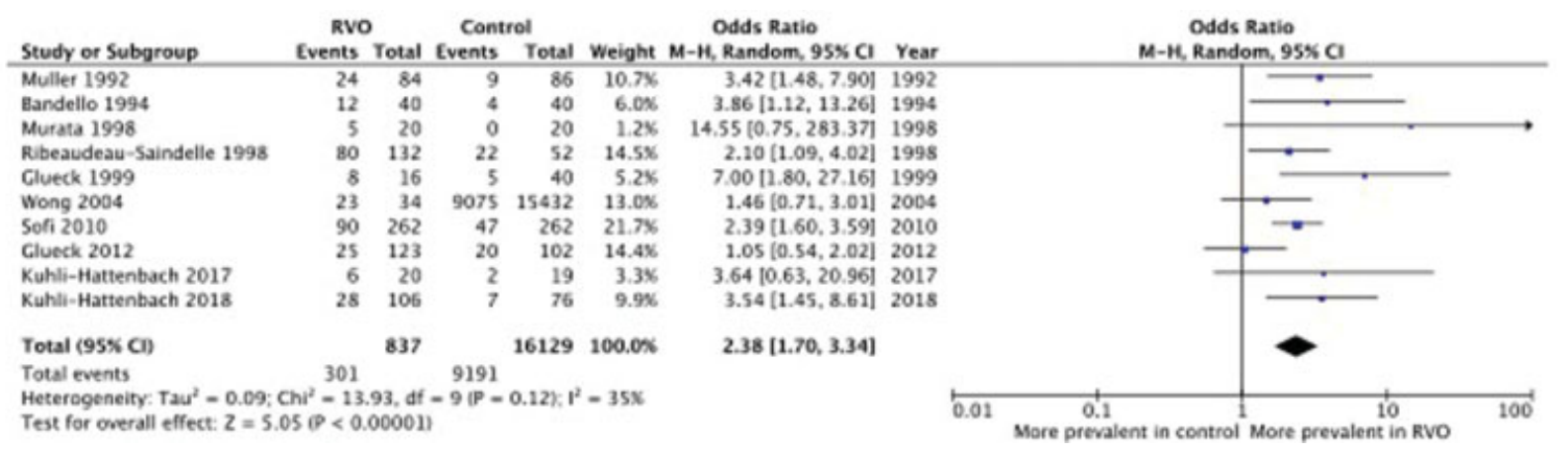

Fig. 2 Prevalence of RVO in subjects with abnormal $L p(a)$ versus $L p(a)$ within normal range. Forest plot of the studies in which abnormal $L p(a)$ was defined by values above a prespecified upper normal limit. Cl, confidence interval. Lp(a), lipoprotein (a); RVO, retinal vein occlusion.

rather than venous thrombosis because cardiovascular risk factors, instead of thrombophilia, seem to play a pre-eminent role. Our study, reporting the first systematic review and

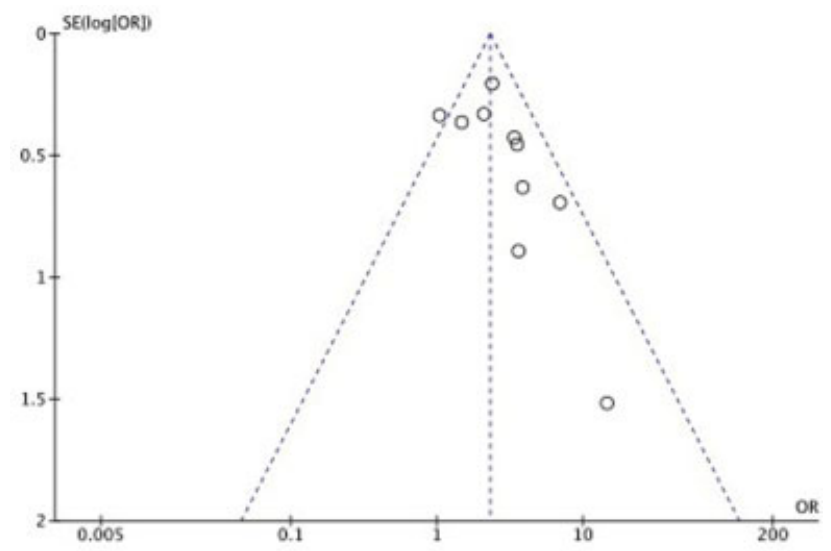

Fig. 3 Funnel plot of the included studies in which abnormal Lp(a) was defined by values above a prespecified upper normal limit. meta-analysis of investigations on the relationship between $\mathrm{Lp}(\mathrm{a})$ levels and RVO, supports the hypothesis that high $\mathrm{Lp}(\mathrm{a})$ is associated with RVO. ${ }^{28-31}$ In fact $\mathrm{Lp}(\mathrm{a})$ levels above upper normal limits associated with RVO and patients with RVO had significantly higher plasma Lp(a) levels than controls.

The pathophysiologic role of $\operatorname{Lp}(\mathrm{a})$ in RVO may be explained by its multiple interactions with vascular and hemostatic homeostasis. Indeed, RVO is to a large extent the consequence of venous stasis provoked by compression from the near atherosclerotic arteriolar wall. ${ }^{40}$ Therefore, differently from other risk factors, such as thrombophilia or hypercholesterolemia which act rather selectively on one of the two vascular beds, $\mathrm{Lp}(\mathrm{a})$ may favor RVO acting on both retinal arterioles and veins by enhancing vascular inflammation and by impairing fibrinolysis, thus favoring thrombosis. This twofold pathogenic activity of $\operatorname{Lp}(\mathrm{a})$ may be especially relevant in a condition like RVO which is on the border between venous and arterial thrombosis. ${ }^{1-5}$

Based on our results, lowering $\mathrm{Lp}(\mathrm{a})$ represents an attractive approach to the prevention of RVO or its recurrence. Currently, the only recommended strategy to reduce $\operatorname{Lp}(\mathrm{a})$ 


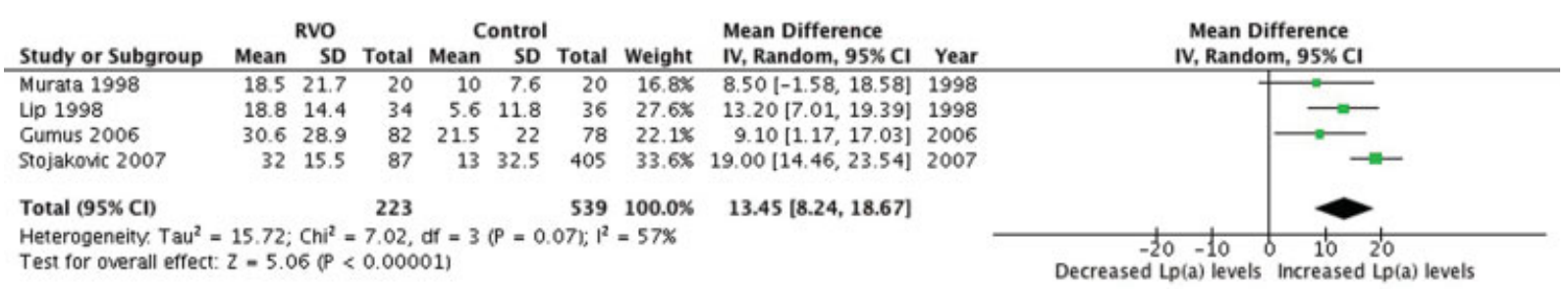

Fig. 4 Forest plot evaluating the WMD in $L p(a)$ levels between patients with RVO and controls. Cl, confidence interval. Lp(a), lipoprotein (a); RVO, retinal vein occlusion; WMD, weighted mean difference.

remains plasma apheresis (expected reduction $60-80 \%$ ) as there are no drugs able to selectively reduce $\operatorname{Lp}(\mathrm{a})$ levels, although some reduction has been reported with aspirin (15-20\%), lomitapide and mipomersen (30\%), PCSK9-inhibitors (30\%), and nicotinic acid (38\%). ${ }^{10}$ However, in the near future lipid nanoparticle-vehiculated short interfering RNAs, such as antisense antiapo(a)oligonucleotides, are expected to revolutionize $\mathrm{Lp}(\mathrm{a})$ lowering therapy. ${ }^{41}$ Indeed, in a recent phase II randomized placebo-controlled trial, the subcutaneous administration of the antisense oligonucleotide anti apo(a) AKCEA-APO(a)-Lrx significantly and dose dependently lowered $\mathrm{Lp}(\mathrm{a})$ plasma levels, with a maximum reduction of $80 \%$ at 6 months. ${ }^{42,43}$ If this approach will obtain approval for clinical use, then its testing in patients with a previous RVO to prevent recurrence or in patients at high risk of RVO to prevent its occurrence will deserve to be assessed.

Our meta-analysis has some limitations. The first is the observational nature of all the included studies which, compared with randomized trials, may make the calculation of a single summary estimate of effect of exposure, in this case high Lp(a) levels, misleading. ${ }^{44,45}$ However, the use of random effects model reduced this risk taking into account the possible variance among studies. ${ }^{45}$ In addition, it is well established that $\mathrm{Lp}(\mathrm{a})$ levels may be influenced by several conditions, such as smoking and diabetes, which may act as confounding factors ${ }^{9}$ and cardiovascular risk-matched selection of control was performed just in one of the studies included, thus limiting the possibility of considering these factors in the analysis. Moreover, we detected the likely presence of publication bias. Nevertheless, this was attenuated by the inclusion in our systematic review of gray literature and not published evidence in commercial publications. ${ }^{46}$ Furthermore, a subgroup analysis according to site of occlusion (BRVO vs. CRVO) was not performed because separate information for these two types of RVO were not provided in the included studies, therefore a possible differential influence of $\mathrm{Lp}(\mathrm{a})$ on BRVO versus CRVO could not be excluded. In fact, some previous studies exploring the impact of other cardiovascular risk factors on RVO have shown that hypertension, peripheral arterial disease, diabetes mellitus, and atherosclerosis are significantly more associated with BRVO than with CRVO. ${ }^{47,48}$ Therefore, further studies addressing the role of $L p(a)$ specifically in BRVO versus CRVO are highly warranted. HRVO, which is considered a third entity by some authors, ${ }^{49}$ was reported only in one study ${ }^{22}$ and may thus be underrepresented. Additionally, the cut-off values and laboratory methods used for the measurement of $\operatorname{Lp}(\mathrm{a})$ varied widely among the included studies and this may have affected the strength of the association between $\operatorname{Lp}(a)$ and RVO. This bias will be overcome only with the standardization of the measurement methods. Finally, despite the non-negligible number of included studies, the number of enrolled patients in our analysis was not large, however, still remarkable representing the largest collection of RVO cases related to $\mathrm{Lp}(\mathrm{a})$ levels reported so far.

\section{Conclusion}

RVO remains an incompletely understood thrombotic disorder with many unsolved questions. To date, no obviously effective treatment is available, and several patients still develop blindness or severe visual impairment. Our data suggest that $\mathrm{Lp}(\mathrm{a})$ may represent an important factor in the pathogenesis of RVO and should be included among parameters to assess when evaluating the risk of RVO or RVO recurrence. Future prospective studies aimed to evaluate the role of $L p(a)$ in RVO risk and recurrence and the effect of $\operatorname{Lp}(\mathrm{a})$-lowering treatments in patients with RVO is highly warranted.

\section{What is Known about This Topic?}

- Retinal vein occlusion(RVO) pathogenesis is still unclear.

- Increased lipoprotein (a) $[\operatorname{Lp}(a)]$ has been associated with both arterial and venous thromboembolism.

- Some studies have shown higher $\operatorname{Lp}(\mathrm{a})$ levels in patients with RVO.

\section{What This Paper Add?}

- Systematic review and meta-analysis analyzing available evidence on the role of $\mathrm{Lp}(\mathrm{a})$ in RVO.

- Increased $\operatorname{Lp}(\mathrm{a})$ is associated with RVO incidence.

- Lp(a) levels are higher in RVO than non RVO subjects.

- Hypothesis-generating information for future clinical trials evaluating $\operatorname{Lp}(\mathrm{a})$ lowering drugs in RVO.

\section{Funding}

This work was supported in part by a grant from Fondazione CARIPLO (\#2018-0483) to P.G.

Conflict of Interest

None declared. 


\section{References}

1 Sivaprasad S, Amoaku WM, Hykin PRVO Guideline Group. The Royal College of Ophthalmologists Guidelines on retinal vein occlusions: executive summary. Eye (Lond) 2015;29(12): 1633-1638

2 Garcia-Horton A, Al-Ani F, Lazo-Langner A. Retinal vein thrombosis: the Internist's role in the etiologic and therapeutic management. Thromb Res 2016;148:118-124

3 Romiti GF, Corica B, Borgi M, et al. Inherited and acquired thrombophilia in adults with retinal vascular occlusion: a systematic review and meta-analysis. J Thromb Haemost 2020;18 (12):3249-3266

4 Ponto KA, Scharrer I, Binder $\mathrm{H}$, et al. Hypertension and multiple cardiovascular risk factors increase the risk for retinal vein occlusions: results from the Gutenberg Retinal Vein Occlusion Study. J Hypertens 2019;37(07):1372-1383

$5 \mathrm{Wu}$ CY, Riangwiwat T, Limpruttidham N, et al. Association of retinal vein occlusion with cardiovascular risk factors: a systematic review and meta-analysis. Retina 2019;39(09):1635-1645

6 Ageno W, Cattaneo R, Manfredi E, et al. Parnaparin versus aspirin in the treatment of retinal vein occlusion. A randomized, double blind, controlled study. Thromb Res 2010;125(02):137-141

7 Tsimikas S. A test in context: lipoprotein(a): diagnosis, prognosis, controversies, and emerging therapies. J Am Coll Cardiol 2017;69 (06):692-711

8 Reyes-Soffer G, Ginsberg HN, Ramakrishnan R. The metabolism of lipoprotein (a): an ever-evolving story. J Lipid Res 2017;58(09): $1756-1764$

9 Nordestgaard BG, Langsted A. Lipoprotein (a) as a cause of cardiovascular disease: insights from epidemiology, genetics, and biology. J Lipid Res 2016;57(11):1953-1975

10 Paciullo F, Momi S, Gresele P. PCSK9 in haemostasis and thrombosis: possible pleiotropic effects of PCSK9 inhibitors in cardiovascular prevention. Thromb Haemost 2019;119(03): 359-367

11 Stulnig TM, Morozzi C, Reindl-Schwaighofer R, Stefanutti C. Looking at $\mathrm{Lp}(\mathrm{a})$ and related cardiovascular risk: from scientific evidence and clinical practice. Curr Atheroscler Rep 2019;21(10): 37

12 Willeit P, Ridker PM, Nestel PJ, et al. Baseline and on-statin treatment lipoprotein(a) levels for prediction of cardiovascular events: individual patient-data meta-analysis of statin outcome trials. Lancet 2018;392(10155):1311-1320

13 Sharrett AR, Ballantyne CM, Coady SA, et al; Atherosclerosis Risk in Communities Study Group. Coronary heart disease prediction from lipoprotein cholesterol levels, triglycerides, lipoprotein(a), apolipoproteins A-I and B, and HDL density subfractions: the Atherosclerosis Risk in Communities (ARIC) Study. Circulation 2001;104(10):1108-1113

14 Kouvari M, Panagiotakos DB, Chrysohoou C, et al. Lipoprotein (a) and 10-year cardiovascular disease incidence in apparently healthy individuals: a sex-based sensitivity analysis from ATTICA cohort study. Angiology 2019;70(09):819-829

15 Kronenberg F, Kronenberg MF, Kiechl S, et al. Role of lipoprotein (a) and apolipoprotein(a) phenotype in atherogenesis: prospective results from the Bruneck study. Circulation 1999;100(11): $1154-1160$

16 Ariyo AA, Thach C, Tracy R. Cardiovascular Health Study Investigators. $\mathrm{Lp}(\mathrm{a})$ lipoprotein, vascular disease, and mortality in the elderly. N Engl J Med 2003;349(22):2108-2115

17 Keil JE, Loadholt CB, Weinrich MC, Sandifer SH, Boyle E Jr. Incidence of coronary heart disease in blacks in Charleston, South Carolina. Am Heart J 1984;108(3 Pt 2):779-786

18 Rajecki M, Pajunen P, Jousilahti P, Rasi V, Vahtera E, Salomaa V. Hemostatic factors as predictors of stroke and cardiovascular diseases: the FINRISK ' 92 Hemostasis Study. Blood Coagul Fibrinolysis 2005;16(02):119-124
19 Lakka HM, Lakka TA, Tuomilehto J, Sivenius J, Salonen JT. Hyperinsulinemia and the risk of cardiovascular death and acute coronary and cerebrovascular events in men: the Kuopio Ischaemic Heart Disease Risk Factor Study. Arch Intern Med 2000;160(08): 1160-1168

20 Dentali F, Gessi V, Marcucci R, Gianni M, Grandi AM, Franchini M. Lipoprotein(a) as a risk factor for venous thromboembolism: a systematic review and meta-analysis of the literature. Semin Thromb Hemost 2017;43(06):614-620

21 Kunutsor SK, Mäkikallio TH, Kauhanen J, Voutilainen A, Laukkanen JA. Lipoprotein(a) is not associated with venous thromboembolism risk. Scand Cardiovasc J 2019;53(03):125-132

22 Kuhli-Hattenbach C, Miesbach W, Lüchtenberg M, Kohnen T, Hattenbach LO. Elevated lipoprotein (a) levels are an independent risk factor for retinal vein occlusion. Acta Ophthalmol 2017;95 (02):140-145

23 Bandello F, Viganò D’Angelo S, Parlavecchia M, et al. Hypercoagulability and high lipoprotein(a) levels in patients with central retinal vein occlusion. Thromb Haemost 1994;72(01):39-43

24 Murata M, Saito T, Takahashi S, Ichinose A. Plasma lipoprotein(a) levels are high in patients with central retinal artery occlusion. Thromb Res 1998;91(04):169-175

25 Sofi F, Marcucci R, Fedi S, et al. High lipoprotein (a) levels are associated with an increased risk of retinal vein occlusion. Atherosclerosis 2010;210(01):278-281

26 Stojakovic T, Scharnagl H, März W, Winkelmann BR, Boehm BO, Schmut O. Low density lipoprotein triglycerides and lipoprotein (a) are risk factors for retinal vascular occlusion. Clin Chim Acta 2007;382(1-2):77-81

27 Wong TY, Larsen EK, Klein R, et al. Cardiovascular risk factors for retinal vein occlusion and arteriolar emboli: the Atherosclerosis Risk in Communities \& Cardiovascular Health studies. Ophthalmology 2005;112(04):540-547

28 Kuhli-Hattenbach C, Hellstern P, Miesbach W, Kohnen T, Hattenbach LO. Prevalence of elevated lipoprotein (a) levels in patients $<60$ years of age with retinal vein occlusion. Klin Monatsbl Augenheilkd 2018;235(01):81-86

29 Ribeaudeau-Saindelle F, Glacet-Bernard A, Lelong F, Coscas G, Soubrane G. Retinal vein occlusion and lipoprotein (a). J Fr Ophtalmol 1998;21(04):245-250

30 Müller HM, Diekstall FF, Schmidt E, März W, Canzler H, Demeler U. Lipoprotein (a): a risk factor for retinal vascular occlusion. Ger J Ophthalmol 1992;1(05):338-341

31 Glueck CJ, Bell H, Vadlamani L, et al. Heritable thrombophilia and hypofibrinolysis. Possible causes of retinal vein occlusion. Arch Ophthalmol 1999;117(01):43-49

32 Glueck CJ, Hutchins RK, Jurantee J, Khan Z, Wang P. Thrombophilia and retinal vascular occlusion. Clin Ophthalmol 2012;6:1377-1384

33 Gumus K, Kadayifcilar S, Eldem B, et al. Is elevated level of soluble endothelial protein $C$ receptor a new risk factor for retinal vein occlusion? Clin Exp Ophthalmol 2006;34(04):305-311

34 Lip PL, Blann AD, Jones AF, Lip GY. Abnormalities in haemorheological factors and lipoprotein (a) in retinal vascular occlusion: implications for increased vascular risk. Eye (Lond) $1998 ; 12(\mathrm{Pt}$ 2):245-251

35 Rosen L. An intuitive approach to understanding the attributable fraction of disease due to a risk factor: the case of smoking. Int J Environ Res Public Health 2013;10(07):2932-2943

36 Wan X, Wang W, Liu J, Tong T. Estimating the sample mean and standard deviation from the sample size, median, range and/or interquartile range. BMC Med Res Methodol 2014;14:135

37 Kontopantelis E, Reeves D. MetaEasy: a meta-analysis add-in for Microsoft Excel. J Stat Softw 2009;30:1-25

38 Wells GA, Shea B, O'Connell D, et al. The Newcastle-Ottawa Scale (NOS) for assessing the quality if nonrandomized studies in metaanalyses. 2012. Accessed September 12, 2020 at: http://wwwohrica/programs/clinical_epidemiology/oxfordasp 
e302 Increased Lipoprotein (a) and Retinal Vein Occlusion Paciullo et al.

39 Peters JL, Sutton AJ, Jones DR, Abrams KR, Rushton L. Comparison of two methods to detect publication bias in meta-analysis. JAMA 2006;295(06):676-680

40 Prisco D, Marcucci R. Retinal vein thrombosis: risk factors, pathogenesis and therapeutic approach. Pathophysiol Haemost Thromb 2002;32(5-6):308-311

41 Langsted A, Nordestgaard BG. Antisense oligonucleotides targeting lipoprotein(a). Curr Atheroscler Rep 2019;21(08):30

42 Jia X, Liu J, Mehta A, Ballantyne CM, Virani SS. Lipid-lowering biotechnological drugs: from monoclonal antibodies to antisense therapies-a clinical perspective. Cardiovasc Drugs Ther 2020 (e-pub ahead of print). Doi: 10.1007/s10557-02007082-X

43 Tsimikas S, Karwatowska-Prokopczuk E, Gouni-Berthold I, et al; AKCEA-APO(a)-LRx Study Investigators. Lipoprotein(a) reduction in persons with cardiovascular disease. N Engl J Med 2020;382 (03):244-255
44 Blettner M, Sauerbrei W, Schlehofer B, Scheuchenpflug T, Friedenreich $\mathrm{C}$. Traditional reviews, meta-analyses and pooled analyses in epidemiology. Int J Epidemiol 1999;28(01):1-9

45 Stroup DF, Berlin JA, Morton SC, et al. Meta-analysis of observational studies in epidemiology: a proposal for reporting. Metaanalysis Of Observational Studies in Epidemiology (MOOSE) group. JAMA 2000;283(15):2008-2012

46 Paez A. Gray literature: an important resource in systematic reviews. J Evid Based Med 2017;10(03):233-240

47 Cho B-J, Bae SH, Park SM, et al. Comparison of systemic conditions at diagnosis between central retinal vein occlusion and branch retinal vein occlusion. PLoS One 2019;14(08):e0220880

48 Bucciarelli P, Passamonti SM, Gianniello F, Artoni A, Martinelli I. Thrombophilic and cardiovascular risk factors for retinal vein occlusion. Eur J Intern Med 2017;44:44-48

49 Karia N. Retinal vein occlusion: pathophysiology and treatment options. Clin Ophthalmol 2010;4:809-816 